\title{
Développement d'un piégeur passif pour la surveillance du tritium atmosphérique
}

\author{
P. Caldeira Ideias ${ }^{1, *}$, O. Pierrard ${ }^{1}$, D. Tournieux ${ }^{1}$, G. Manificat ${ }^{1}$ et L. Tenailleau ${ }^{2}$ \\ ${ }^{1}$ Institut de radioprotection et de sûreté nucléaire, PRP-ENV/SESURE/LS3E, 31, rue de l'Écluse, BP 40035, 78116 Le Vésinet, France. \\ ${ }^{2}$ Groupe d'études atomiques de la marine nationale, EAMEA - BCRM - CC19, 50115 Cherbourg-Octeville, France.
}

Reçu le 13 janvier 2016 / Accepté le 4 novembre 2016

\begin{abstract}
Résumé - Le développement d'un dispositif passif de prélèvement du tritium atmosphérique a été initié en 2012 pour optimiser les moyens de surveillance régulière de l'environnement en complémentarité avec les dispositifs existants (barboteur, condenseur). Le concept devait répondre à des contraintes de simplicité de mise en œuvre sur le terrain (pas d'apport en énergie, compacité...) et présenter des performances équivalentes aux meilleurs dispositifs disponibles actuellement sur le marché. L'étude a porté sur le piégeage du tritium sous forme de vapeur d'eau dans l'air (HTO). Elle a intégré le design du piège de terrain, la sélection des matériaux utilisés, le développement d'une chambre à atmosphère contrôlée et d'un système de désorption des pièges passifs pour des analyses en laboratoire. Une série de tests de qualifications dans des conditions de laboratoire puis dans l'environnement a été menée. Les résultats obtenus avec ces prélèvements passifs démontrent une très bonne concordance avec ceux obtenus avec les moyens utilisés habituellement en routine (barboteurs, condenseurs) sur une plage d'activité couvrant le bruit de fond naturel jusqu'à des niveaux identiques à ceux des rejets gazeux des installations nucléaires. Ils démontrent en outre le potentiel offert par ce nouveau dispositif pour une utilisation opérationnelle en surveillance des rejets, de l'environnement mais aussi pour des études spécifiques de type cartographique ou une utilisation en situation de crise.
\end{abstract}

Keywords : passive / sampling / tritium / HTO / environmental / surveillance crisis / molecular sieve / tritium bubbler / condenser

\begin{abstract}
The development of an atmospheric tritium monitoring system based on passive sampling launched in 2012 to improve the environmental monitoring capabilities to complement conventional means. The purpose of this project was to design a passive technical solution that provides a compact system with no external power supply needed and having performances equivalent to the best devices currently available on the market. The study focused on trapping tritium in the form of water vapor in the air (HTO). This project included the design of the device, the selection of trapping materials, the development of an atmospheric control chamber and a desorption system for the passive traps laboratory analyzes. Tests were conducted in laboratory and then in the environment. The results obtained with the passive samplers show a very good correlation with those obtained with the traditional means (bubblers and vapor condensers) on a range of activities covering the natural background and going up to levels similar to those of gaseous discharges from nuclear installations. This new passive device shows a very good potential for use in radioactive releases monitoring, for environmental surveillance but also for specific studies (such as mapping) and emergency situations.
\end{abstract}

\section{Introduction}

Le tritium est omniprésent dans l'environnement, qu'il soit produit naturellement ou qu'il soit rejeté par l'industrie civile

\footnotetext{
$\overline{\text { *Auteur de correspondance }}$ : pedro.caldeiraideias@irsn.fr
}

ou par des installations dédiées aux applications militaires (Belot et al., 1996) (Fig. 1). En France, une trentaine d'installations nucléaires produisent et rejettent du tritium sous trois formes différentes : gaz tritié ( $\left.\mathrm{HT}, \mathrm{T}_{2}\right)$, eau tritiée (HTO, $\mathrm{T}_{2} \mathrm{O}$ ) ou sous la forme de composés organiques volatiles (ex : $\mathrm{CTH}_{3}$ ). Avec le carbone 14, le tritium fait partie des radionucléides les plus rejetés par les installations nucléaires 


\section{Rejets atmosphériques en ${ }^{3} \mathrm{H}$ en 2014 (TBq)}

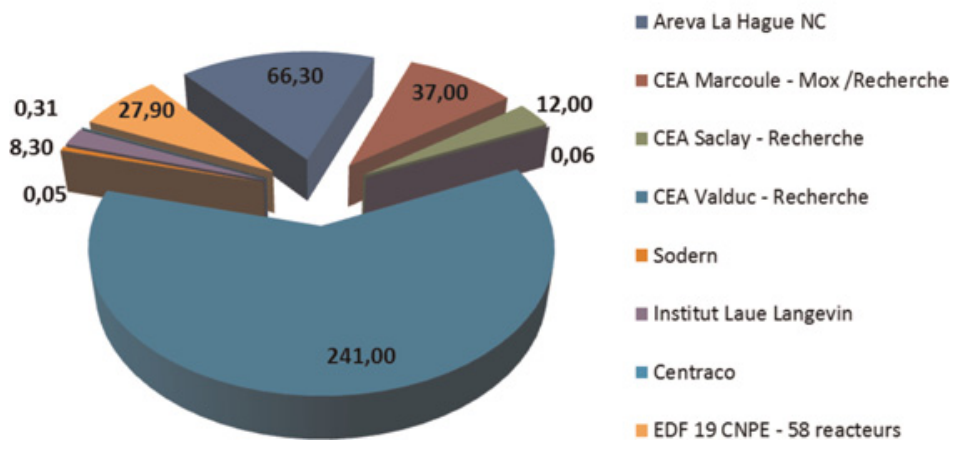

Fig. 1. Rejets gazeux en tritium pour l'année de 2014 (correspondance masse activité : $1 \mathrm{~g} \Leftrightarrow 360 \mathrm{TBq}$ ).

Fig. 1. Gaseous tritium releases in 2014.

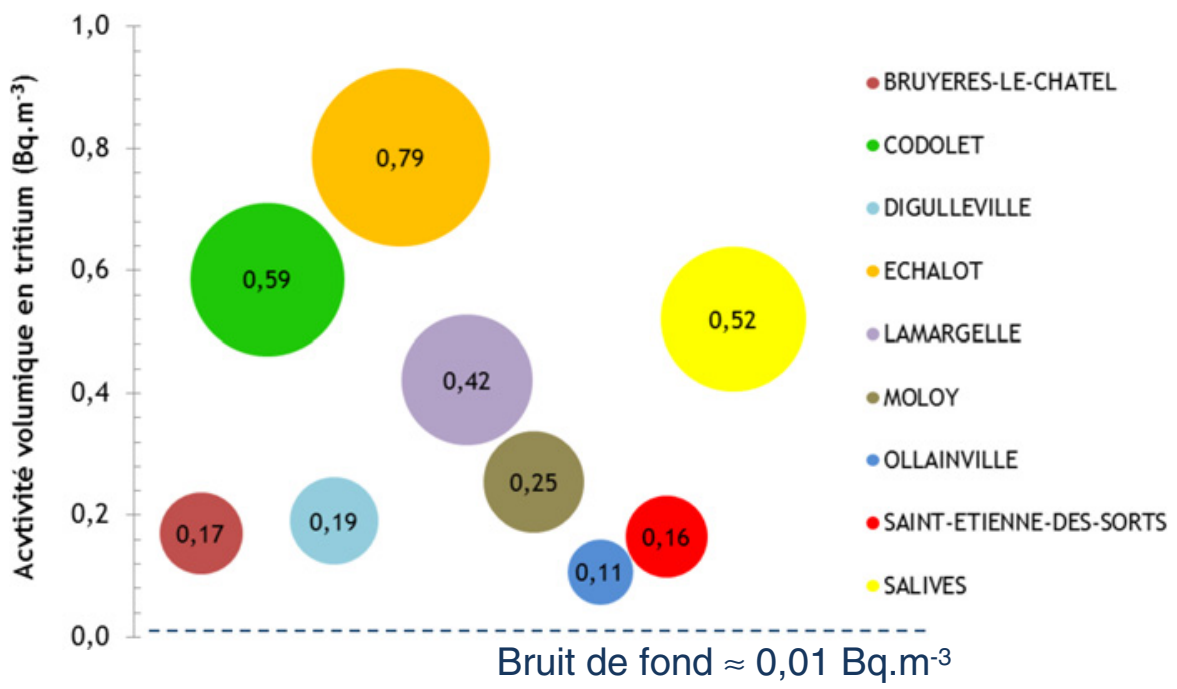

Fig. 2. Moyennes des activités volumiques significatives en tritium (Bq. $\mathrm{m}^{-3}$ ) mesurées dans l'environnement français à proximité d'installations nucléaires en 2012-2014.

Fig. 2. Tritium average significant activity $\left(\mathrm{Bq} . \mathrm{m}^{-3}\right)$ measured in French nuclear facilities environment. Source: RNM.

(Le Guen, 2008). Dans le cadre de la surveillance réglementaire imposée aux exploitants d'installations nucléaires, plusieurs dizaines de milliers de mesures de tritium sont réalisées chaque année par l'ensemble des acteurs dans les rejets et dans tous les compartiments de l'environnement. La mesure de ces radionucléides dans l'atmosphère (gaz, eaux de pluie) a une importance primordiale pour asseoir une surveillance pertinente des rejets des installations, comprendre les transferts de ce radionucléide dans l'environnement et in fine les impacts écologiques et sanitaires. (Calmet et al., 2002).

Pour répondre à leurs besoins de contrôles, de surveillance et d'études, les différents acteurs de la surveillance, notamment les exploitants nucléaires, doivent se doter de moyens de surveillance atmosphérique de ce radionucléide pour différentes gammes d'activité. Si la mesure directe du tritium est relativement développée pour la surveillance des rejets aux cheminées (généralement une chambre d'ionisation), les niveaux d'activités dans l'atmosphère extérieure sont trop faibles pour permettre d'envisager cette solution technique(Belovodski et al., 1996; Caldeira Ideias, 2014). La surveillance environnementale du tritium dans l'air repose aujourd'hui en France sur la réalisation de prélèvements par barboteurs, et plus récemment par des condenseurs, suivie d'analyses par scintillation liquide en laboratoire (Guetat et al., 2008; Caldeira Ideias, 2014). La Figure 2 synthétise les activités volumiques moyennes en tritium mesurées dans l'air, au-dessus des seuils de décision, déclarées au Réseau national de mesures de la radioactivité de l'environnement (RNM) dans la période 2012 à 2014.

L'objectif de cette étude était de développer une technique passive pour piéger la vapeur d'eau tritiée atmosphérique (HTO) en vue d'aboutir à un dispositif simple d'installation, permettant d'effectuer des prélèvements sur des durées variables sans effet de dilution du tritium dans l'eau (exemple système de barbotage). Ce dispositif a été conçu, développé et testé en laboratoire sur une plateforme dimensionnée spécifiquement pour le tritium sur la forme HTO. 


\section{Méthode et matériel}

\subsection{Concepts pour l'échantillonnage passif de l'air dans l'environnement}

Le principe du piégeage passif repose sur la différence de concentrations établie entre le milieu à échantillonner et le média de piégeage (Lida et al., 1995; Otlet et al., 2005). Cet état est maintenu tant que l'adsorbant (support de piégeage) n'est pas saturé. Pour le dimensionnement d'un dispositif basé sur ce principe, il est essentiel de maîtriser les variables suivantes : état du contaminant à prélever, caractéristiques physico-chimiques de l'adsorbant, temps de prélèvement et configuration géométrique du préleveur.

\subsection{Principes d'adsorption des gaz}

Les molécules superficielles d'un liquide présentent des hétérogénéités qui génèrent des liaisons chimiques à l'origine des phénomènes de capillarité et de tension superficielle. Les mêmes phénomènes sont observés dans le cas d'un solide. L'hétérogénéité de la matière implique que les solides ont une capacité à capter les gaz qui se trouvent dans leurs environs. De ce fait, la concentration d'une substance de la phase gazeuse s'équilibre avec la concentration de cette substance à la surface du solide, c'est le phénomène d'adsorption (Dawoud et Aristov, 2002). La caractéristique la plus intéressante d'un adsorbant est sa surface d'échange. Elle dépend de sa porosité. Les forces en présence dans ce phénomène intermoléculaire peuvent être regroupées en deux groupes : les forces de van der Waals et les forces chimiques ioniques (Suzuki, 1990 ; Kohl et Nielsen, 1997). On parle alors de physisorption et de chimisorption respectivement. La théorie qui explique ce phénomène a été proposée par Langmuir : la surface des adsorbants présente des espaces pouvant être occupés par des molécules adsorbables. Quand une molécule atteint un pore, elle s'y insère et se rend non échangeable avec l'atmosphère. Un retour à l'atmosphère peut être opéré par réaction endothermique. Selon la théorie de Langmuir, l'adsorption se ferait en une seule couche. Cependant, il est reconnu que les molécules peuvent créer une seconde couche par formation d'un champ de force de van der Waals, puis une troisième et ainsi de suite (Suzuki, 1990). Les forces responsables de la plupart des phénomènes d'adsorption sont donc les forces de van der Waals. Cette prédominance implique une adsorption plus importante pour les gaz qui présentent de faibles pressions de vapeur (Kohl et Nielsen, 1997). En conséquence, les gaz les plus lourds sont adsorbés préférentiellement, tout comme ceux qui ont une température critique moins élevée. D'autres caractéristiques, comme la saturation du mélange gazeux et la polarité de la molécule, ont une influence importante dans le processus d'adsorption. En l'absence d'altération de la structure chimique des molécules adsorbées et de l'adsorbant, le phénomène d'adsorption est complètement réversible. L'adsorbant peut être complètement régénéré après récupération de l'adsorbat. L'adsorption est accompagnée d'une libération d'énergie équivalente à la chaleur latente de condensation (Dawoud et Aristov, 2002; Sun et Besant, 2005). La quantité de gaz adsorbée est dépendante de la concentration du gaz à échantillonner dans l'air. Elle dépend aussi de la température et de la pression à laquelle la réaction se produit. Une autre variable très importante est la capacité de piégeage de l'adsorbant utilisé. Cette capacité dépend de la composition chimique, mais aussi d'autres propriétés intrinsèques au matériau telles que : la distribution granulométrique des particules composant l'adsorbant, la taille des pores, la surface spécifique et les courbes isothermes d'adsorption.

\subsection{Média de piégeage}

Un tamis moléculaire a été retenu et préféré à du gel de silice car cet adsorbant concentre plusieurs qualités très intéressantes, notamment son faible taux de rémanence (phénomène de conservation et de relargage $a$ posteriori) de tritium pour les niveaux d'activités attendus dans l'environnement. Les tamis moléculaires sont constitués de zéolithes, qui sont composés de structures cristallines d'aluminosilicates formées d'unités tétraédriques comme les nids d'abeilles. Les tamis moléculaires possèdent des caractéristiques d'adsorption extrêmement sélectives. Ils ont des pores de dimension déterminée à la fabrication suivant le diamètre des molécules à sélectionner. Les molécules avec un diamètre supérieur ne pourront pas atteindre les surfaces internes des nids d'abeilles par exclusion. Le tamis moléculaire utilisé dans cette étude est de type $13 \times\left(\mathrm{Na}_{86}\left[\left(\mathrm{AlO}_{2}\right)_{86}\left(\mathrm{SiO}_{2}\right)_{106}\right] \times \mathrm{H}_{2} \mathrm{O}\right)$ avec une taille des pores de $10^{-9} \mathrm{~m}$.

Les tamis moléculaires ont une grande capacité d'adsorption même pour de faibles quantités d'adsorbat recherchées (Paulus et al., 2002). Ils présentent aussi une grande affinité pour les substances non-saturées (liaisons chimiques multiples) et polaires. Les tamis moléculaires possèdent très peu d'eau dans leur structure moléculaire et ne s'effritent pas quand l'eau adsorbée est éliminée. De ce fait, ils peuvent être chauffés à plus de $400^{\circ} \mathrm{C}$ tout en maintenant leur structure.

\subsection{Dimensionnement du piégeur passif de tritium}

Le dimensionnement et l'exploitation des préleveurs passifs de tritium demandent non seulement la connaissance de la variable extensive que représente l'abondance des différentes espèces chimiques présentes dans le gaz, notamment la vapeur à piéger, mais aussi la connaissance des principales variables thermodynamiques intensives : la température $(\mathrm{T})$ et la pression $(\mathrm{Pa})$. Dans cette étude, le comportement de $\mathrm{HTO}$ est assimilé à celui de $\mathrm{H}_{2} \mathrm{O}$ par simplification, considérant qu'il n'y a pas de différence significative décrite dans la littérature entre les deux molécules (Whicker et al., 2011). Un flux diffusif de la vapeur d'eau dans l'air vers l'adsorbant est créé lorsque l'adsorbant est exposé à l'air. Tout système thermodynamique tendant vers une situation d'équilibre, l'enjeu est d'obtenir une relation simple et proportionnelle entre l'eau fixée à la surface de l'adsorbant et celle de l'atmosphère. Ce flux diffusif est régi par la loi de Fick. La diffusivité d'un composant $\mathrm{X}$ dans un mélange de substances, dépend du flux de l'ensemble des composants de ce mélange. Il serait nécessaire de connaître les flux des différents composants de l'air (ex. gaz rares, dioxyde de carbone, méthane) pour calculer toutes les diffusivités. Cependant une approximation permet de simplifier les calculs de diffusivité en considérant la diffusion d'un seul composant dans un mélange stationnaire, principe qui s'applique à la 


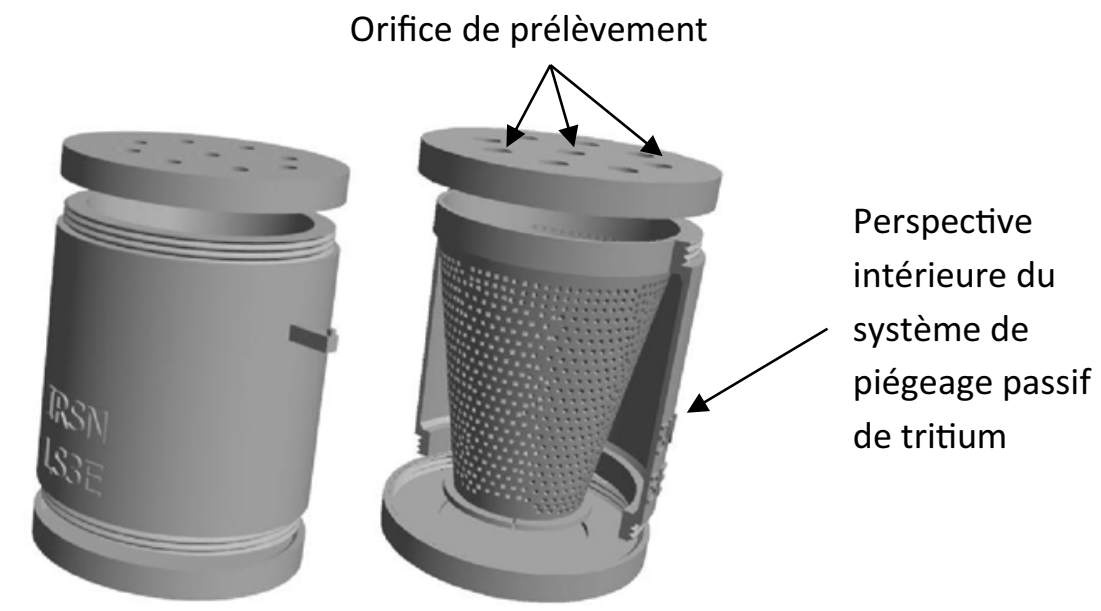

Fig. 3. Vues intérieure et extérieure du dispositif de prélèvement passif.

Fig. 3. Inside and outside views of the tritium passive sampling device.

vapeur d'eau. Nous pourrons donc considérer que la vapeur d'eau se déplace de l'atmosphère vers le préleveur passif par diffusion moléculaire. À température et pression constantes, et dans des conditions laminaires, le flux d'eau unidirectionnel peut-être décrit par l'équation suivante :

$$
F_{\mathrm{H}_{2} \mathrm{O}}\left[\frac{m o l}{m^{2} \cdot s}\right]=-D_{\mathrm{H}_{2} \mathrm{O}}\left[\frac{m^{2}}{s}\right] \times \frac{\partial C_{\mathrm{H}_{2} \mathrm{O}}}{\partial z}\left[\frac{m o l}{m^{4}}\right]
$$

où $F_{\mathrm{H}_{2} \mathrm{O}}$ est le flux d'eau, $D_{\mathrm{H}_{2} \mathrm{O}}$ est le coefficient de diffusion moléculaire de l'eau dans l'air, $C_{\mathrm{H}_{2} \mathrm{O}}$ est la concentration de la vapeur d'eau et « $z$ » est la longueur du parcours de diffusion.

Le tamis moléculaire est une substance très hydrophile. Sans introduire un moyen de maîtriser le flux d'eau de l'atmosphère vers le piégeur passif, l'adsorbant serait saturé avant la fin de la période de prélèvement considéré. De façon empirique, une géométrie a été trouvée qui permet de réaliser le prélèvement dans des conditions d'absorption de vapeur d'eau pratiquement linéaires. Ce qui permet d'avoir pendant toute la durée de l'échantillonnage un prélèvement représentatif de la moyenne de la teneur en vapeur d'eau de l'air. Pour optimiser le prélèvement, de nombreuses géométries ont été testées. Celle qui favorise le prélèvement dans les conditions de l'environnement est la géométrie tronconique (Fig. 3). La forme tronconique optimise les échanges entre l'air et l'adsorbant tout en garantissant que l'adsorbant ne se sature pas sur les durées de prélèvement entre 24 heures et un mois. En réalité il existe un front d'adsorption qui avance plus au moins vite dans les directions axiale et longitudinale, en fonction du temps. Par comparaison avec les moyens classiques de prélèvement, dans ce système passif la pompe est remplacée par le pouvoir d'adsorption du tamis moléculaire et le débitmètre par le couvercle et la géométrie tronconique intérieure (Fig. 3).

\subsection{Préparation, prélèvement et récupération du prélèvement de la vapeur d'eau tritiée}

Les conditions initiales d'utilisation des adsorbants comme média de piégeage sont fondamentales. Ainsi, pour s'assurer que le prélèvement ne sera pas dilué ou marqué, il faut procéder en amont à la purification ou la régénération du matériau adsorbant, ainsi qu'à son stockage sous atmosphère sèche.

Lors de l'exposition du piégeur passif dans l'atmosphère à échantillonner, celui-ci va prélever l'eau $\left(\mathrm{H}_{2} \mathrm{O}\right)$, l'eau tritiée (HTO) et dans une proportion beaucoup moins importante d'autres gaz présents, comme le dioxyde de carbone $\left(\mathrm{CO}_{2}\right)$. Simultanément, un hygromètre mesure et enregistre l'humidité relative et la température de l'air durant toute la durée de prélèvement

À la fin du prélèvement, le piégeur est envoyé vers le laboratoire où la récupération du tritium à doser est effectuée à l'aide d'un système de désorption. La désorption est un phénomène endothermique, qu'est effectuée en chauffant le tamis moléculaire à $400^{\circ} \mathrm{C}$ sous un flux de $10 \mathrm{~L} \cdot \mathrm{h}^{-1}$ d'azote. En faisant circuler ce gaz à travers l'adsorbant durant la période de chauffe, on maintient une pression partielle de l'adsorbat $\left(\mathrm{HTO}+\mathrm{H}_{2} \mathrm{O}\right)$ dans le flux d'azote inférieure à la pression d'équilibre de l'adsorbat sur la surface du solide. La vapeur d'eau tritiée piégée est ainsi libérée et envoyée vers un système de piège froid. Pour assurer une condensation maximale, le piège froid, constitué d'un mélange de $50 \%$ d'eau échangé et $50 \%$ de glycol est maintenu à $-20^{\circ} \mathrm{C}$. Ensuite $10 \mathrm{ml}$ de liquide scintillant sont ajoutés à $10 \mathrm{ml} \mathrm{du}$ prélèvement d'eau qui sont récupérés puis comptés par scintillation liquide.

Pour avoir un échantillon représentatif et fiable, des précautions préalables doivent être prises au laboratoire pour : a) empêcher la dilution ou la contamination du prélèvement par l'atmosphère ; b) maitriser la rémanence de l'eau tritiée dans le tamis moléculaire (pour les activités en tritium élevées) ; c) prendre en compte un possible fractionnement isotopique entre $\mathrm{HTO}$ et $\mathrm{H}_{2} \mathrm{O}$ dans la phase condensée. Les points a) et b) sont maîtrisés en s'assurant du suivi du poids de l'adsorbant après régénération et à la fin du prélèvement. Selon la littérature Whicker et al. (2011) mentionnent dans ces travaux un fractionnement isotopique entre la phase condensée et la phase gazeuse (point $\mathrm{c}$ ) de $<1 \%$ (pour une désorption à $200^{\circ} \mathrm{C}$ ). Pour minimiser ce phénomène il est essentiel de maintenir le piège froid à une température de $-20^{\circ} \mathrm{C}$. 


\subsection{Calcul de l'activité en tritium dans l'atmosphère}

Afin de déterminer l'activité volumique du tritium dans l'air, un calcul de l'hygrométrie absolue doit être effectué à partir des données d'hygrométrie relative et de température enregistrées durant le prélèvement en suivant les étapes (Matos et al., 1998) :

Calcul de la pression de vapeur d'eau :

$$
P_{\mathrm{v}, \text { saturation }}=e^{a_{0}+a_{1} \cdot T_{\mathrm{air}}(K)+\frac{a_{2}}{T_{\mathrm{air}}(K)}+a_{3} \cdot T_{\mathrm{air}}(K)^{2}+a_{4} \cdot T_{\mathrm{air}}(K)^{3}} .
$$

Les coefficients $a_{0}, a_{1}, a_{2}, a_{3}, a_{4}$ sont données par Matos et al. (1998).

Calcul de la pression de vapeur de l'eau :

$$
P_{\mathrm{v}}=\frac{H R(\%)}{100} \times P_{\mathrm{v}, \text { saturation }} .
$$

Calcul de l'humidité absolue :

$$
\begin{aligned}
Y_{\mathrm{H}_{2} \mathrm{O}} & =0,622 \times \frac{P_{\mathrm{v}}}{P_{\text {atmosphérique }}-P_{\mathrm{v}}} \times \frac{P_{\text {air }} \cdot M_{\text {air }}}{R \cdot T_{\text {air }}} . \\
& =0,622 \times \frac{P_{\mathrm{v}}}{P_{\text {atmosphérique }}-P_{\mathrm{v}}} \times \rho_{\text {air }} .
\end{aligned}
$$

L'activité mesurée dans l'échantillon suite à un comptage par scintillation liquide, exprimée en becquerel par litre est retranscrite en becquerel par mètre cube d'air humide, par intégration de l'hygrométrie absolue selon la formule suivante :

$$
A_{\text {tritium, air }}=A_{\text {tritium, échantillon }} \times Y_{\mathrm{H}_{2} \mathrm{O}},
$$

Avec :

$A_{\text {tritium, air }}=$ Activité de la vapeur d'eau tritiée dans l'air $\left(\right.$ Bq. $\mathrm{m}^{-3}$ )

$A_{\text {tritium, échantillon }}=$ Activité de la vapeur d'eau tritiée de l'air condensé $\left(\mathrm{Bq} . \mathrm{L}^{-1}\right)$

$Y_{\mathrm{H}_{2} \mathrm{O}}=$ Quantité d'eau par mètre cube d'air $\left(\mathrm{g} . \mathrm{m}^{-3}\right)$

$A=$ Activité radioactive $(\mathrm{Bq})$

$a_{0}, a_{1}, a_{2}, a_{3}, a_{4}=$ Coefficients empiriques

$\mathrm{HR}=$ Humidité relative $(\%)$

$M_{\mathrm{i}}=$ Masse molaire du composant i $\left(\mathrm{g} \cdot \mathrm{mol}^{-1}\right)$

$N=$ Nombre de moles $(\mathrm{mol})$

$P_{\text {atmosphérique }}=$ Pression atmosphérique dans le calcul de l'humidité absolue $(\mathrm{Pa})$

$P_{\mathrm{v}}=$ Pression de vapeur $(\mathrm{Bar})$

$P_{\mathrm{v}}$, saturation $=$ Pression de vapeur de saturation (Bar)

$P_{\text {air }}=$ Pression atmosphérique au moment de la mesure $(\mathrm{Pa})$

$R=$ Constante des gaz parfaits $\left(\mathrm{Pa}^{3} \mathrm{~m}^{3} \cdot \mathrm{mol}^{-1} \cdot \mathrm{K}^{-1}\right)$

$T=$ Température $\left({ }^{\circ} \mathrm{K}\right)$

$V=$ Volume $\left(\mathrm{m}^{3}\right)$

$\rho=$ Masse volumique $\left(\mathrm{kg} \cdot \mathrm{m}^{-3}\right)$.

\subsection{Développement d'une plateforme d'études de la vapeur d'eau tritiée atmosphérique}

Un système nommé PETRA (Plateforme d'études du tritium atmosphérique) de régulation de l'humidité relative a été développé afin de caractériser précisément les préleveurs passifs. Ce procédé, bien que non-adiabatique, réduit
Tableau 1. Caractéristiques opérationnelles et techniques des préleveurs passifs de tritium.

Table 1. Operational and technical characteristics of tritium passive samplers.

$\begin{array}{ll}\text { Caractéristiques du tamis moléculaire } & \\ \text { Quantité de tamis moléculaire } & 180 \mathrm{~g} \\ \text { Capacité de piégeage maximal } & 30 \% \mathrm{en} \mathrm{poids} \\ \text { Densité apparente du tamis moléculaire } & 643,7 \mathrm{~g} . \mathrm{dm}^{-3} \\ \quad \begin{array}{l}\text { Distribution granulométrique (classe }>95 \%) \\ \text { Dimensions des préleveurs passifs }\end{array} & 1,6-2,5 \mathrm{~mm} \\ \quad \begin{array}{l}\text { Diamètre, hauteur } \\ \text { Paramètres opérationnels }\end{array} & 94 \mathrm{et} 111 \mathrm{~mm} \\ \quad \begin{array}{l}\text { Temps d'opération } \\ \text { Température d'opération } \\ \text { Hygrométrie relative }\end{array} & 24 \mathrm{~h} \text { à } 30 \text { jours } \\ & -10 \text { à } 35{ }^{\circ} \mathrm{C} \\ & 30-100 \%\end{array}$

fortement l'influence de la température, variable importante de l'hygrométrie relative qui conditionne l'adsorption des gaz (Suzuki, 1990). Le schéma de fonctionnement du procédé de régulation de l'humidité relative de l'air injecté dans la plateforme est composé de trois ensembles. La première partie du système consiste à assécher l'air ambiant, en le faisant passer dans une colonne remplie de gel de silice. La deuxième partie comprend une unité de barbotage de l'air sec dans une solution avec une activité en tritium connu. La troisième partie est constituée d'une boite à gants de $300 \mathrm{~L}$ qui contient l'atmosphère généré. Le débit d'air est de 8 à $10 \mathrm{~L} \cdot \mathrm{min}^{-1}$ et il est assuré par une pompe à vide qui aspire l'air de l'extérieur vers la colonne et le bac contenant l'activité en tritium souhaité. Le débit d'air sec et d'air humide est contrôlé par un système piloté par ordinateur à partir des données acquises par plusieurs sondes hygrométriques. Ce système permet donc de maîtriser l'activité volumique en tritium dans la boîte à gants et de disposer d'un environnement contrôlé pour qualifier les dispositifs passifs. Ce système travail à la pression atmosphérique et permet de générer une atmosphère avec une valeur d'humidité relative qui varie entre $0 \pm 1 \%$ et $90 \pm 5 \%$.

\section{Résultats et discussion}

\subsection{Paramètres}

Les caractéristiques opérationnelles et techniques des préleveurs passifs de tritium qui ont été développées, sont présentées dans le Tableau 1.

\subsection{Représentativité du prélèvement}

La représentativité du prélèvement a été étudiée en quantité d'eau prélevée et non pas en mètres cubes d'air prélevés. La linéarité de prélèvement de la vapeur d'eau atmosphérique par les piégeurs passifs a été analysée avec une variation d'humidité, de la surface d'échange entre l'atmosphère et le préleveur passif par le dimensionnement des couvercles (Fig. 3). L'objectif est de démontrer que le préleveur passif garde une relation de linéarité quand le taux de prélèvement et l'humidité changent. Les données sont représentées sur la Figure 4. Les tests ont été effectués avec 
une variation de l'humidité relative entre $50 \pm 5 \%$ (piégeurs : $\mathrm{B}, \mathrm{C}, \mathrm{D}$ ) et $90 \pm 5 \%$ (piégeurs : $\mathrm{A}, \mathrm{E}$ ) pour une température de $22 \pm 3{ }^{\circ} \mathrm{C}$ dans PETRA. L'expérience a été accomplie en faisant varier le taux de prélèvement et en suite le niveau d'humidité. Les taux de prélèvements obtenus pour les piégeurs passifs sont en moyenne de : $\mathrm{A}=2,03 \pm 0,08 \mathrm{~g}$ d'eau/ jour ; $\mathrm{B}=0,76 \pm 0,11 \mathrm{~g}$ d'eau/jour ; $\mathrm{C}=0,83 \pm 0,07 \mathrm{~g}$ d'eau/jour ; $\mathrm{D}=0,99 \pm 0,05 \mathrm{~g}$ d'eau/jour ; $\mathrm{E}=0,34 \pm 0,01 \mathrm{~g}$ d'eau/jour. Les pièges $\mathrm{A}$ et $\mathrm{B}$ ont été installés respectivement sous une atmosphère à $90 \pm 5 \%$ et à $50 \pm 5 \%$ d'humidité relative. Les taux de prélèvement ont varié en pourcentage de masse d'eau prise de : $\mathrm{A}=4 \%$ et $\mathrm{B}=14 \%$. Les variations plus importantes observées dans les taux de prélèvements, notamment pour le préleveur $\mathrm{B}$, sont dues à une variation de l'humidité de $\pm 5 \%$ dans le système PETRA. Les piégeurs passifs $\mathrm{A}$ et $\mathrm{B}$ présentaient une surface d'échange égale. Il a été vérifié que le taux de prélèvement est proportionnel mais moins élevé quand l'humidité est moins importante. Les piégeurs B, C et D disposaient des taux de prélèvements différents. Ils ont été exposés à une atmosphère de $50 \pm 5 \%$ d'humidité et $22 \pm 3{ }^{\circ} \mathrm{C}$

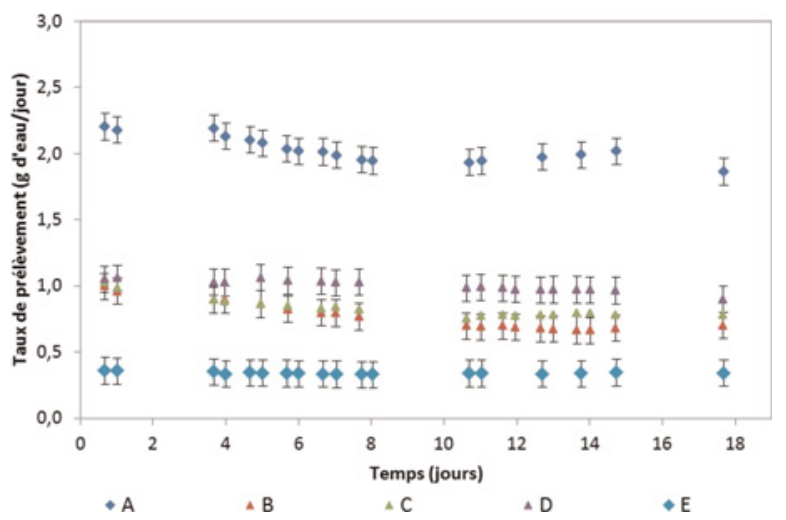

Fig. 4. Représentation des résultats des taux de prélèvements des piégeurs passifs tritium sous différentes conditions d'humidité : $50 \pm 5$ $\%$ (piégeurs : B, C, D) et $90 \pm 5 \%$ (piégeurs : A, E).

Fig. 4. Results of the sampling rate of tritium passive samplers for different humidity conditions: $50 \pm 5 \%$ (samplers: $B, C, D$ ) and $90 \pm 5 \%$ (samplers: A, E).

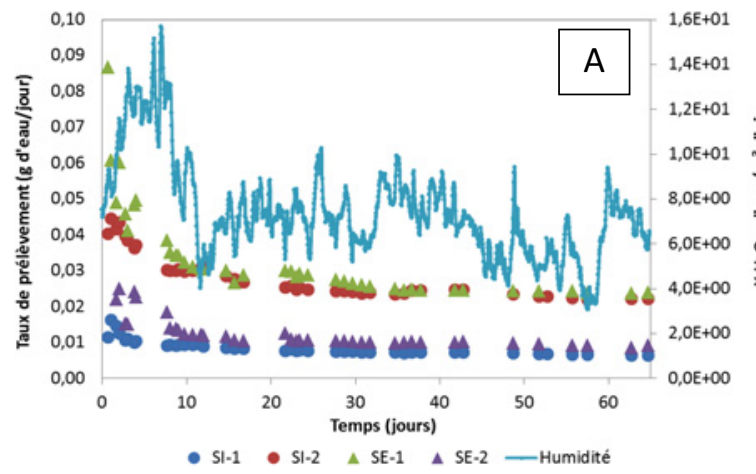

de température. Il a été vérifié que les taux de prélèvements ont eu une variation maximal de $\mathrm{B}=14 \% ; \mathrm{C}=8 \%$ et $\mathrm{D}=5 \%$. Les taux de prélèvements restent proportionnels au niveau d'hygrométrie dans PETRA en faisant varier aussi bien la surface d'échange que l'humidité relative. Le piégeur A et $\mathrm{E}$ ont été exposés à une atmosphère de $90 \pm 5 \%$. Le piégeur $\mathrm{A}$ présentait une surface d'échange six fois plus importante que le E. Nous avons pu constater que le taux de prélèvement du dispositif $\mathrm{A}$ est approximativement six fois supérieures à celui du E. Le piégeur E présentait une variation du taux de prélèvement de $3 \%$. Les tests effectués ont eu pour but de démontrer qu'avec une hygrométrie stable, moyennant les incertitudes, que le taux de prélèvement reste stable. Ce système passif présent un taux de prélèvement d'autant plus élevé que la quantité de vapeur d'eau dans l'air est importante. Cette expérience permet de démontrer que le temps de prélèvement peut-être raccourci ou rallongé en faisant simplement varier la surface d'échange entre l'atmosphère et le piégeur.

Le taux de prélèvement des piégeurs passifs a aussi été étudié dans l'environnement. Les données observées sont illustrées sur la Figure 5A. Cette figure représente le taux de prélèvement des pièges pour des conditions variables d'humidité dans l'atmosphère en fonction du temps. Il a été vérifié que le taux de prélèvement accompagne la variation de l'hygrométrie. Les taux de prélèvements sont élevés au début et ils tendent vers une stabilisation quand la variation de l'hygrométrie est moins importante. La garantie de la représentativité du prélèvement a ainsi pu être vérifiée (Fig. 5A). Les différents taux de prélèvements observés correspondent à des vitesses de prélèvements distinctes qui découlent de la variabilité de l'hygrométrie dans l'atmosphère. La variabilité du taux de prélèvement est de $10 \%$ en moyenne. La reproductibilité du taux de prélèvement est présentée sur la Figure 5B. Le coefficient de variation entre les cinq piégeurs passifs pour chaque point de prélèvement est $0,7 \%(\mathrm{CV}=$ écart type/moyenne $)$.

\subsection{Performance de prélèvement}

Des tests de reproductibilité ont été effectués sous atmosphère contrôlé, pour trois niveaux d'activités en tritium différents. Les gammes d'activités varient entre 6 et

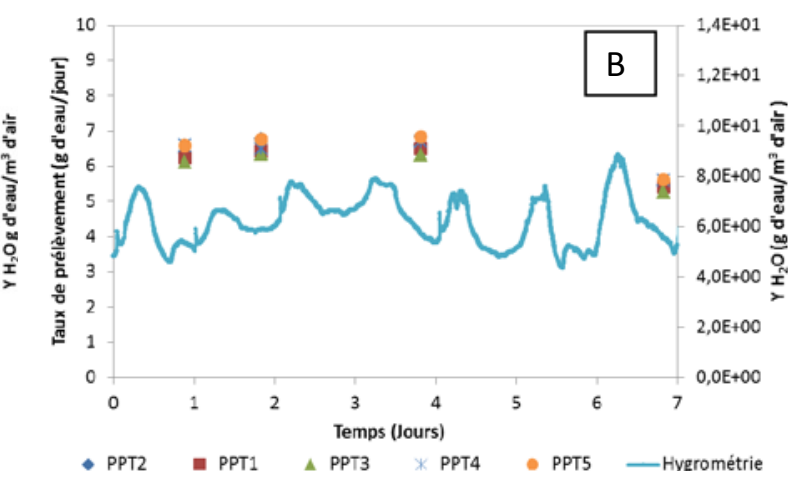

Fig. 5. Représentation graphique de la réponse des préleveurs passifs dans l'environnement (SE-1, SE-2, SE-3 représentent des piégeurs avec des taux de prélèvement différents ; PPT1, PPT2, PPT3, PPT4, PPT5 représentent des prélèvements effectués avec le même taux de prélèvement).

Fig. 5. Representation of the passive samplers performance in the environment (SE-1, SE-2, SE-3 represents the different passive samplers with different sampling rates; PPT1, PPT2, PPT3, PPT4, PPT5 represents different passive samplers with the same sampling rate). 
Tableau 2. Reproductibilité de l'activité en tritium dans la vapeur d'eau piégée par les préleveurs passifs pour différents niveaux d'activités. Table 2. Reproducibility of the tritium sampling devices for different levels of activity.

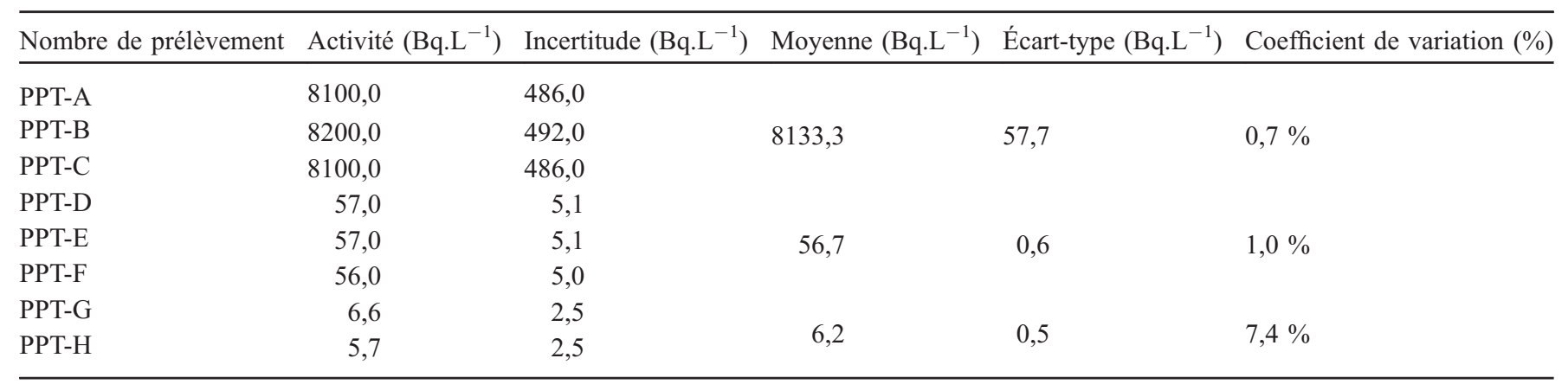

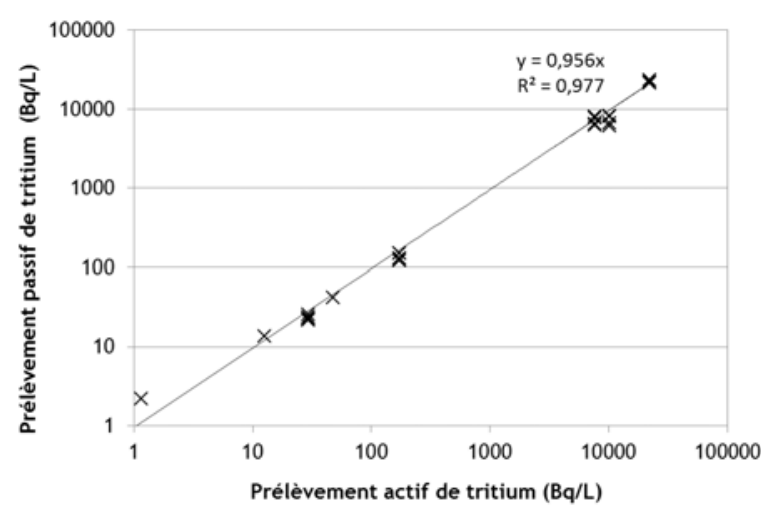

Fig. 6. Rapport entre l'activité en tritium prélevée avec les pièges passifs et l'activité prélevée avec les moyens actifs (barboteur et H3R7000 ${ }^{\circledR}$ ), en laboratoire et dans l'environnement.

Fig. 6. Ratio of tritium sampled with passive and active methods (bubbling and $H 3 R 7000^{\mathbb{R}}$ ) in laboratory and environment conditions.

8200 Bq.L ${ }^{-1}$ d'eau de condensation récupérée. Les résultats sont résumés dans le Tableau 2. Les coefficients de variation calculés sur l'activité en tritium prélevé avec la technique de prélèvement passif pour chaque point varient entre 0,7 et $7,4 \%$, la technique est donc considérée comme reproductible.

Afin d'évaluer la performance des préleveurs passifs, des séries de prélèvements ont été réalisés en parallèle avec des moyens actifs (barboteur et H3R7000 ${ }^{\circledR}$ ), considérés ici comme des méthodes de référence, pour différents niveaux d'activités dans une gamme couvrant le niveau environnemental à des niveaux équivalents à ceux mesurés régulièrement dans les effluents atmosphériques des installations nucléaires. Les résultats de cette série de mesures sont résumés sur la Figure 6. Les activités obtenues après récupération du prélèvement varient entre 3 et $25000 \mathrm{~Bq}$. $\mathrm{L}^{-1}$ d'eau récupérée. Le coefficient de corrélation est $\mathrm{R}^{2}=0,977$ et la pente de la courbe ajusté est de 0,956 . Les résultats montrent une très bonne concordance des activités mesurées entre les dispositifs passif et actif standards. Pour les faibles niveaux de tritium, jusqu'à $100 \mathrm{~Bq} . \mathrm{L}^{-1}$ d'eau de condensation, il a été vérifié que la technique de désorption développé récupère $>99 \%$ du tritium piégé en une seule désorption. Pour les activités de tritium plus importantes, $>1000 \mathrm{~Bq} . \mathrm{L}^{-1}$ il a été vérifié un phénomène de rémanence de $5 \%$. Valeur inférieure à la valeur de l'incertitude de la mesure par scintillation liquide (incertitude relative $\mathrm{k}=2$ de 6 à $8 \%$, pour un temps de comptage de 1000 minutes).

\section{Conclusion}

Le dispositif développé par l'Institut de Radioprotection et de Sureté Nucléaire (IRSN) pour le prélèvement passif du tritium atmosphérique sous forme HTO permet d'obtenir des résultats reproductibles et au moins équivalents aux moyens classiques de prélèvement actif par barbotage dans l'eau ou par condensation. La plage d'utilisation du piégeur passif est potentiellement très importante, allant des niveaux d'activités proches du bruit de fond naturel jusqu'à des niveaux de l'ordre des rejets des installations nucléaires (plus de $10 \mathrm{kBq} \cdot \mathrm{m}^{-3}$ ). Les bonnes performances, le faible encombrement, le faible coût et l'absence d'alimentation énergétique positionnent le piégeur passif comme un dispositif d'avenir pour répondre aux besoins de la surveillance de l'environnement, en situation normale comme en cas de crise. La simplicité de cette technologie permettra d'assurer la surveillance de l'environnement et de réaliser des études radiologiques pertinentes pour un coût modique, mais surtout elle ouvre de nouvelles possibilités, comme celle de pouvoir réaliser des études spatiales sur le marquage de l'environnement par les rejets chroniques proches d'une installation nucléaire. Ce dispositif de prélèvement passif de tritium a fait objet d'un dépôt de brevet, référencé sous le $n^{\circ}$ WO2014037461, consultable sur le site de l'Institut National de la Propriété Industrielle.

Des nouveaux axes de développement et de recherche seront menés sur le piégeage passif d'hydrogène tritiée (HT) et celui $\mathrm{du}{ }^{14} \mathrm{C}$ sous forme de dioxyde de carbone, ainsi que sur la compréhension de l'influence dans le taux de prélèvement de la vitesse du vent et de l'influence de la couche limite.

\section{Références}

Belot Y, Roy M, Métivier H. 1996. Le tritium, de l'environnement à l'homme. Les Éditions de Physiques, IPSN.

Belovodski L, Gaevoy F, Golubev A, Kosheleva T. 1996. Tritium oxide wash-out by drops. J. Environ. Radioactivity 36(2-3): 129-139.

Caldeira Ideias P. 2014. Prélèvement du tritium atmosphérique. In: Rapport IRSN PRP/SESURE/2014-24.

Calmet D, et al. 2002. État des travaux normatifs dans le domaine du mesurage des radionucléides dans l'environnement. Radioprotection 37: 41-58. 
Dawoud B, Aristov Y. 2002. Experimental study on the kinetics of water vapor sorption on selective water sorbents, silica gel and alumina under typical operation conditions of sorption heat pumps. Int. J. Heat Mass Transfer 46: 273-281.

Grosse AV, Johnston WM, Wolfgang RL, Libby WF. 1951. Tritium in nature, Research Institute of Temple University, Philadelphia, Pennsylvania, Institute of Nuclear Studies and Department of Chemistry, University of Chicago. Science 113: $1-2$.

Guetat P, Douche C, Hubinois JC. 2008. Le tritium et l'environnement : sources, mesures et transferts, CEA, Centre de Valduc. Radioprotection 43: 547-569.

Le Guen B. 2008. Impact du tritium autour des centrales nucléaires EDF. Radioprotection 43(2): 177-191.

Lida T, Yokoyama S, Fukuda H, Ikebe Y. 1995. A simple passive method of collecting water vapor for environmental tritium monitoring. Nucl Technol. Publishing 58: 23-27.
Matos MAA, Pereira FJMA, Tarelho LAC, Loureiro JM. 1998. Propriedades termofisicas de gases. In: 7th International Conference of Chemical Engineering, Portuguese Society of Chemistry, Vol. 1, pp. 105-113.

Otlet RL, Walker AJ, Mather ID. 2005. A compact diffusion sampler for environmental applications requiring HTO or HTO+HT determinations. Fusion Sci. Technol. 48: 366-369.

Paulus LR, Walker DW, Thompson KC. 2002. A comparison of desiccant materials used for monitoring in a semi-arid climate. Health Phys. 85(3): 348-356.

Sun J, Besant RW. 2005. Heat and mass transfer during silica gelmoisture interactions. Int. J. Heat Mass Transfer 48: 4953-4962.

Suzuki M. 1990. Adsorption engineering, Kodansha Ltd Tokyo. USA: Elsevier Science Publishing Company

Whicker JJ, Dewart JM, Allen SP, Eisele WF, McNaughton MW, Green AA. 2011. Corrections for measurements of tritium in subterranean vapor using silica gel. Environ. Monit. Assess.: 135-143.

Citation de l'article : Caldeira Ideias P, Pierrard O, Tournieux D, Manificat G, Tenailleau L. 2017. Développement d'un piégeur passif pour la surveillance du tritium atmosphérique. Radioprotection 52(1): 57-64. 